\title{
SURGICAL TREATMENT OF PULMONARY ARTERY SARCOMA
}

Eckhard Mayer, MD

Jörg Kriegsmann, $\mathrm{MD}^{\mathrm{b}}$

Andreas Gaumann, $\mathrm{MD}^{\mathrm{b}}$

Hans Ulrich Kauczor, MD

Manfred Dahm, MD

Ulrich Hake, MD

Franz Xaver Schmid, MD

Hellmut Oelert, MD ${ }^{\mathrm{a}}$
Objective: Pulmonary artery sarcomas are rare and usually fatal tumors. The diagnosis is difficult and delayed in most cases. Newer imaging techniques could allow early diagnosis in patients with symptoms of pulmonary vascular obstruction. Surgical resection improves clinical symptoms and offers the only chance of cure. We report the case histories of 7 patients with primary pulmonary artery sarcomas treated by surgical resection with or without adjuvant therapy.

Methods: Seven patients (3 women and 4 men; mean age, 52.3 years; preoperative New York Heart Association functional class III/IV, $n=5 / 2$ ) underwent operations. Malignancy was preoperatively suspected in 5 patients, and 2 patients had a presumptive diagnosis of chronic pulmonary embolism. Tumor resection with partial or total prosthetic replacement $(n=2)$, reconstruction $(n=5)$, or both, of central parts of the pulmonary arteries was performed in 6 patients. Thromboendarterectomy was necessary in 4 patients, and pneumonectomy was necessary in 2 patients. Six patients received adjuvant therapy.

Results: There was no perioperative mortality. All patients had a substantial improvement in exercise tolerance and hemodynamics 3 months after their operations. Four patients died 7, 9, 18, and 19 months after their operations because of recurrent tumor or pulmonary metastases. Two patients are alive 21 and 35 months after primary surgical repair, with pulmonary metastases detected by computed tomographic scans. One patient is alive 62 months after resection without clinical or radiologic signs of tumor recurrence or metastasis.

Conclusions: Early diagnosis of primary pulmonary artery sarcomas can be improved by computed tomography and magnetic resonance scanning. Radical surgical resection probably presents the only chance for cure. The role of neoadjuvant or adjuvant treatment modalities has to be defined. Pulmonary artery sarcoma need not necessarily be a fatal diagnosis.

(J Thorac Cardiovasc Surg 2001;121:77-82)
D rimary malignant tumors of the pulmonary artery (PA) are rare. The diagnosis is either delayed or established after death because symptoms are nonspe-

From the Departments for Cardiothoracic and Vascular Surgery, ${ }^{\text {a }}$ Pathology, ${ }^{\mathrm{b}}$ and Radiology, ${ }^{\mathrm{c}}$ Johannes Gutenberg-University, Mainz, Germany.

Received for publication June 6, 2000; revisions requested Aug 1, 2000; revisions received Aug 28, 2000; accepted for publication Aug 31, 2000

Address for reprints: Eckhard Mayer, MD, Department for Cardiothoracic and Vascular Surgery, Johannes Gutenberg University Hospital, Langenbeckstr 1, 55101 Mainz, Germany (E-mail: emayer@mail.uni-mainz.de).

Copyright (C) 2001 by The American Association for Thoracic Surgery

0022-5223/2001 $\$ 35.00+0 \quad \mathbf{1 2 / 1 / 1 1 1 4 2 3}$

doi:10.1067/mtc.2001.111423 cific and survival time is limited. With newer radiologic imaging techniques, earlier identification seems to be feasible. Aggressive surgical treatment improves clinical symptoms and offers the only chance for cure.

Since 1989, a multidisciplinary program for diagnosis and treatment of pulmonary hypertension by thromboendarterectomy or lung transplantation has been established at our institution. ${ }^{1,2}$ Therefore, a significant number of patients with different causes of pulmonary hypertension have been referred for further evaluation and consequent surgical therapy. Between April 1989 and December 1998, 7 patients with primary sarcoma of the PA underwent resection with or without chemotherapy, radiotherapy, or both. We report these cases, including clinical presentation, diagnosis, surgical management, and outcome. 

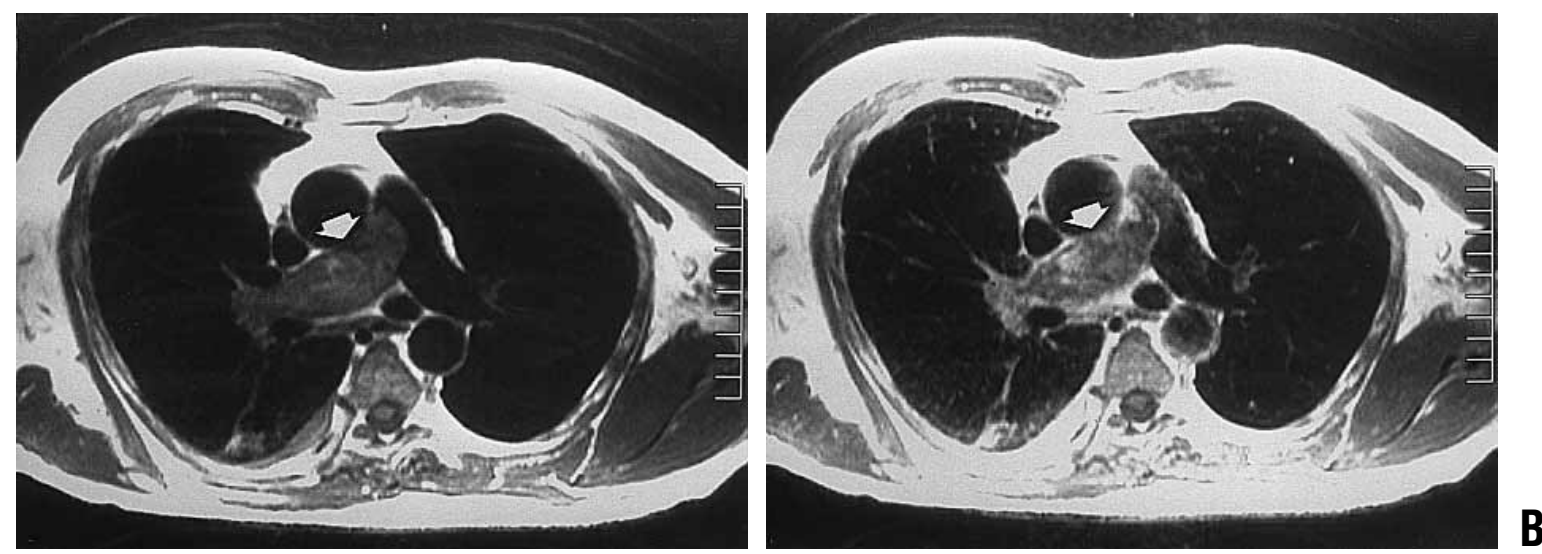

Fig 1. A, MRI scan (patient 7) shows central PA obstruction (arrow). B, Same scan with inhomogeneous enhancement of contrast medium.

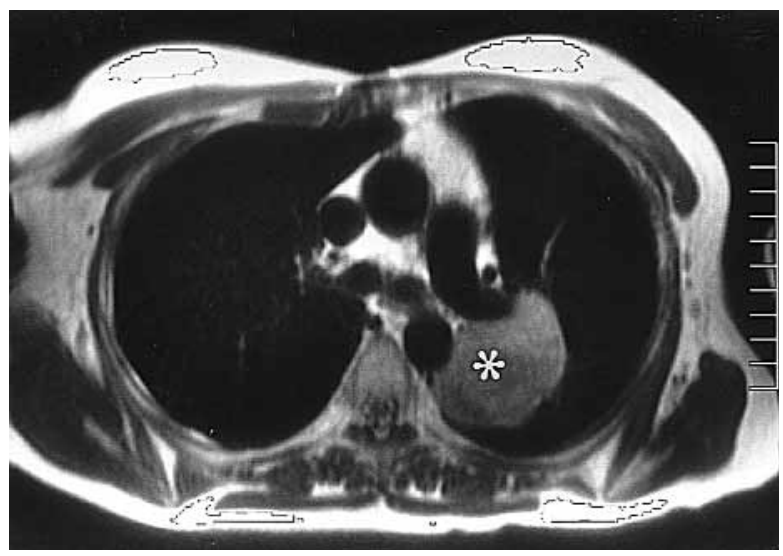

Fig 2. MRI scan (patient 5) showing recurrent pulmonary sarcoma $(*)$ within the left hilum 7 months after pulmonary thromboendarterectomy.

\section{Patients and methods}

From January 1988 to December 1998, 7 patients (3 women and 4 men; mean age, 52.3 years; age range, 31-61 years) were identified. Their symptoms were severe dyspnea (New York Heart Association functional class III/IV, $n=5 / 2$ ), cough $(n=5)$, weight loss $(n=5)$, fatigue $(n=4)$, and chest pain $(n=2)$, with an average duration of symptoms of 6 months (range, 1-16 months). Specific evaluation included computed tomography (CT; $\mathrm{n}=7$ ), magnetic resonance imaging (MRI; $n=3)$, pulmonary angiography $(n=5)$, and echocardiography $(n=6)$. Malignancy was preoperatively suspected in 5 patients on the basis of CT or MRI scanning (Fig 1), and 2 patients had a presumptive diagnosis of subacute or chronic pulmonary embolism.

In 6 patients (patients 1, 2, 3, 4, 6, and 7), the histologic diagnosis of malignancy was established intraoperatively with frozen sections, and the tumor was resected radically with the aid of hypothermic cardiopulmonary bypass $(n=6)$. The completeness of resection was determined by frozen section diagnosis. Tumor resection with partial or total prosthetic replacement $(n=2)$, reconstruction $(n=5)$, or both, of central parts of the pulmonary arteries was performed in 6 patients. In these patients the tumor origin was clearly located or macroscopically suspected at the pulmonary valve or in the central part of the PA. The corresponding areas of the vascular wall were widely resected, followed by partial or total prosthetic replacement. Thromboendarterectomy for intraluminal peripheral tumor growth was necessary in 4 patients (patients 2, 3, 6, and 7), and 2 (patients 3 and 6) patients underwent additional pneumonectomy for histologically proven peripheral tumor spread not safely accessible through thromboendarterectomy techniques, despite periods of circulatory arrest. In 1 patient (patient 7), intraoperative frozen sections revealed a tumor invasion of the aortic root, with peripheral disease within the right PA segmental branches. This patient underwent gross intraluminal tumor resection and main PA reconstruction for palliative restoration of blood flow. One patient (patient 5) underwent pulmonary thromboendarterectomy for suspected subacute pulmonary embolism. Postoperatively, histologic examination of the specimen removed revealed a leiomyosarcoma. This patient was reoperated on after 7 months for recurrence of a central PA tumor (Fig 2) and a large solitary pulmonary metastasis. She underwent right ventricular outflow tract, pulmonary valve, pulmonary trunk, and right PA resection and replacement with polytetrafluoroethylene prostheses and a pulmonary valve homograft combined with a left-sided pneumonectomy. Another patient (patient 6) was reoperated on after 4 months for a solitary pulmonary metastasis by right lower lobe wedge resection (Table I).

One patient (patient 2) did not receive adjuvant therapy, 1 patient (patient 1) received postoperative radiotherapy, 4 patients (patients 4, 5, 6, and 7) had adjuvant chemotherapy, and 1 patient (patient 3 ) had combined chemotherapy and radiotherapy. 


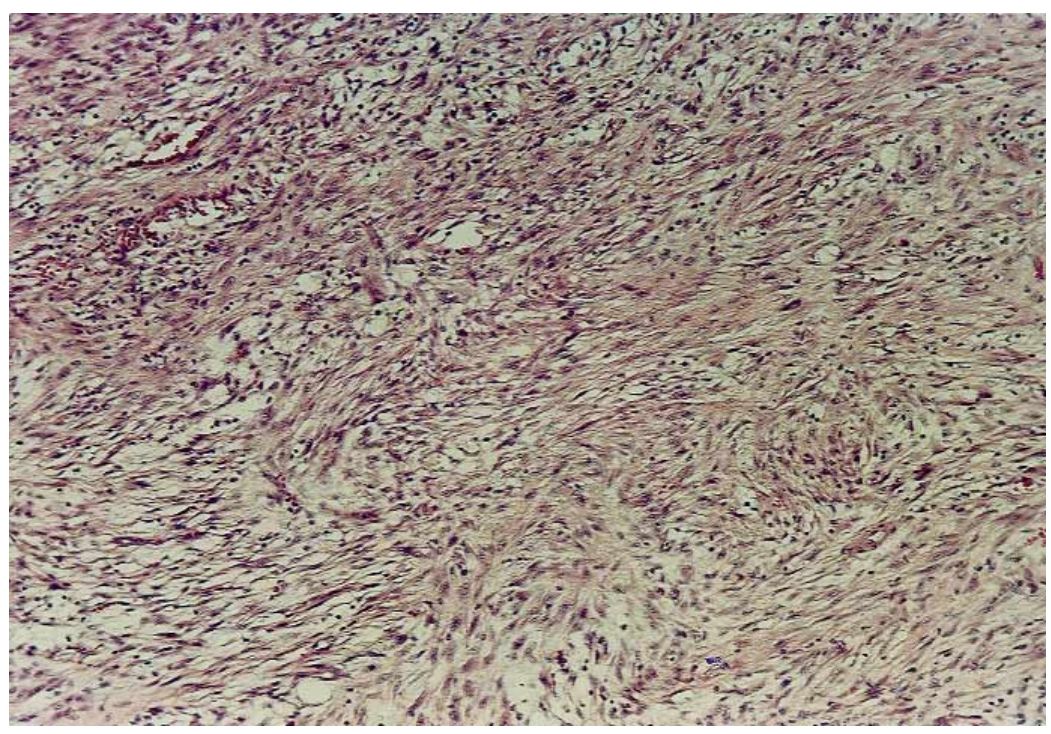

Fig 3. Histology (patient 4): leiomyosarcoma. (Hematoxylin and eosin; original magnification 250x.)

Table I. Patient characteristics and therapeutic procedures

\begin{tabular}{|c|c|c|c|c|c|c|}
\hline Patient No. & $\operatorname{Sex}$ & Age (y) & Surgical procedure & Reoperation & Adjuvant therapy & Survival \\
\hline 1 & M & 56 & Tumor resection, PA reconstruction & & Radiotherapy & Died, 18 mo \\
\hline 2 & $\mathrm{~F}$ & 43 & $\begin{array}{l}\text { Tumor resection, thromboendarterectomy, } \\
\text { PA replacement }\end{array}$ & & None & Died, 9 mo \\
\hline 3 & M & 60 & $\begin{array}{l}\text { Tumor resection, thromboendarterectomy, } \\
\text { right pneumonectomy, PA reconstruction }\end{array}$ & & $\begin{array}{l}\text { Radiotherapy and } \\
\text { chemotherapy }\end{array}$ & Died, 7 mo \\
\hline 4 & M & 31 & $\begin{array}{l}\text { Tumor and right ventricular outflow } \\
\text { tract resection and reconstruction }\end{array}$ & & Chemotherapy & $\begin{array}{l}\text { Alive, } 62 \mathrm{mo} \text {, } \\
\text { without disease }\end{array}$ \\
\hline 5 & $\mathrm{~F}$ & 49 & Thromboendarterectomy & $7 \mathrm{mo}$ & Chemotherapy & Died, 19 mo \\
\hline 6 & M & 61 & $\begin{array}{l}\text { Tumor resection, thromboendarterectomy, } \\
\text { left pneumonectomy, PA replacement }\end{array}$ & $4 \mathrm{mo}$ & Chemotherapy & $\begin{array}{l}\text { Alive, } 35 \text { mo, with } \\
\text { pulmonary metastases }\end{array}$ \\
\hline 7 & M & 60 & Gross tumor resection, thromboendarterectomy & & Chemotherapy & $\begin{array}{l}\text { Alive, } 21 \text { mo, with } \\
\text { pulmonary metastases }\end{array}$ \\
\hline
\end{tabular}

\section{Results}

Intraoperative frozen section diagnosis was achieved in 6 patients. Three patients (patients 1,2, and 4) had negative resection margins, 2 patients (patients 3 and 6) received pneumonectomy on the basis of positive margins, and 1 patient (patient 7) had tumor invasion of the aortic root without possibility of further resection. Final histologic and immunohistochemical examinations showed a myogenous tumor growth pattern in 5 patients, with positive smooth muscle differentiation (desmin) in 5 and smooth muscle actin in 4 patients, representing leiomyosarcomas (Fig 3). The 2 other specimens showed areas resembling malignant fibrous histiocytoma and were classified as intimal sarcomas. Results of the immunohistochemical analysis are shown in Table II.

All patients survived the operation (reoperation), and 2 patients had bleeding complications after pneu- monectomy. However, surgical reintervention for bleeding was not necessary. Three months after the operations, all patients were in New York Heart Association functional class I $(n=4)$ or II $(n=3)$, with a substantial improvement in exercise tolerance and hemodynamics.

Four patients (patients 1, 2, 3, and 5) died at 7, 9, 18, and 19 months after primary operations of recurrent tumor (patients 1,3, and 5) and multiple pulmonary metastases (patients 1 and 2). Three of them (patients 1, 3 , and 5) had radiotherapy, chemotherapy, or both, as an adjuvant treatment. Two patients (patients 6 and 7) are alive 35 and 21 months, respectively, after primary resection and 31 months after reoperation (patient 6). Despite adjuvant chemotherapy, pulmonary metastases (patients 6 and 7) occurred in 2 patients, with no option for further surgical intervention. One patient (patient 4) is alive 62 
Table II. Histology

\begin{tabular}{|c|c|c|c|c|c|c|}
\hline Patient No. & Classification & Vimentin & Desmin & Smooth muscle actin & Cytokeratin & Factor VIII \\
\hline 1 & Leiomyosarcoma & Positive & Positive & Positive & Negative & Negative \\
\hline 2 & Leiomyosarcoma & Positive & Negative & Positive & Negative & Negative \\
\hline 3 & Intimal sarcoma & Positive & Negative & Negative & Negative & Negative \\
\hline 4 & Regressive leiomyosarcoma & Positive & Positive & Positive & Negative & Negative \\
\hline 5 & Leiomyosarcoma & Positive & Positive & Negative & Negative & Negative \\
\hline 6 & Intimal sarcoma plus malignant osteoid & Positive & Negative & Negative & Negative & Negative \\
\hline 7 & Leiomyosarcoma & Positive & Positive & Positive & Negative & Negative \\
\hline
\end{tabular}

months after the operation, without clinical or radiologic (including MRI) signs of tumor recurrence or metastasis (Table I). Overall median survival was 19 months.

\section{Discussion}

Primary tumors of the PA are rare and usually represent sarcomatous neoplasms arising within the intima of the pulmonary trunk ${ }^{3}$ or the bulbus cordis. ${ }^{4}$ The tumor growth in the central PA with additional appositional thrombosis is leading to a progressive obstruction of right ventricular blood flow into the pulmonary circulation. ${ }^{5}$ Emboli to the peripheral pulmonary vasculature may increase right ventricular afterload, resulting in progressive right heart failure. Without surgical intervention, a mean survival time of 1.5 months after diagnosis has been calculated on the basis of 93 cases reported in the literature. ${ }^{6}$

Dyspnea, chest pain, cough, hemoptysis, and syncope are characteristic symptoms of significant pulmonary vascular obstruction. However, in our experience with more than 200 thromboendarterectomy operations for chronic pulmonary embolism since $1989,1,2$ the diagnosis of chronic thromboembolic pulmonary hypertension is often missed or delayed for months or years. It can be speculated that in the majority of patients with fast-growing PA tumors and progressive cardiopulmonary dysfunction, the diagnosis will not be established before death. Therefore, $60 \%$ of the cases reported in the literature have been identified at autopsy. ${ }^{6}$

All our patients had severe and progressive dyspnea for 1 to 16 months before referral to our institution. Multiple clinical and radiologic investigations, including CT, had been performed in all cases. Only in 2 patients was malignancy suspected because of a mediastinal mass detected with CT and an obstructive mass originating from the pulmonary valve detected with transesophageal echocardiography, respectively. These 2 patients were referred for tumor resection. Five patients were referred for surgical therapy of recurrent or chronic pulmonary embolism and underwent a rou- tine preoperative evaluation, including pulmonary angiography, CT, and MRI ( $n=3)$. In 3 of the patients, a malignant intraluminal PA tumor was suspected on the basis of heterogeneous contrast medium enhancement in CT or MRI. The preoperative diagnosis in the remaining 2 patients was chronic thromboembolic pulmonary hypertension. In conclusion, 4 of 5 intraluminal PA obstructions and 1 mediastinal mass originating from the main PA were preoperatively suggested to be malignant. Compared with the literature reports, this is a rather favorable rate of adequate preoperative diagnosis that can be explained by a significant experience with patients with chronic pulmonary embolism and the corresponding CT and MRI findings at our institution. ${ }^{7,8}$ Furthermore, it is likely that chest physicians with a single case experience of PA sarcoma will always include this differential diagnosis when more patients are referred for chronic thromboembolic disease, as is the case at our institution.

Several reports suggest that CT and MRI may be the most useful modalities for differentiation between tumor and thrombotic material. ${ }^{7-10}$ After application of gadolinium-diethylene-triamine-pentaacetic acid, the heterogenous enhancement demonstrated by MRI is characteristic of a vascularized tumor. In rare cases a pedunculated mass arising from the right ventricular outflow tract, pulmonary valve, or pulmonary trunk demonstrated by echocardiography or MRI may be pathognomonic for sarcoma, as in our patient 4 , in whom the tumor was detected with echocardiography at an early stage. Therefore, we consider CT and MRI scanning, combined with transthoracic echocardiography, as sufficient measures for differentiation between tumor and thrombus. Furthermore, a significant experience with diagnosis of chronic thromboembolic pulmonary hypertension seems to be necessary in this rare entity. Preoperative pathologic diagnosis for a better planning of the surgical approach can be achieved by using endovascular catheter biopsy. ${ }^{11}$ However, because appositional thrombotic material covering the tumor is frequent, the sensitivity of the biopsy proce- 
dure has to be evaluated. It is also unlikely that pulmonary angiography is a reliable method for differentiation between thrombus and tumor, whereas pressure gradients between the right ventricular outflow tract and the PA trunk may be helpful in establishing the diagnosis because significant gradients in the central PA are unlikely in thromboembolic disease.

Although fewer than 130 cases have been reported in the literature, a wide variety of the histopathologic pattern of the tumors could be found. Most sarcomas of the PA are luminal sarcomas, which are usually poorly differentiated or undifferentiated and have been called intimal sarcomas. Three of 13 luminal PA sarcomas examined by Burke and Virmani ${ }^{12}$ did not fit under the classification of intimal sarcomas and were identified as a luminal angiosarcoma, an undifferentiated mural sarcoma, and a mural leimyosarcoma. A myxoid background was present in 8 intimal sarcomas, and 3 displayed malignant osteoid. In our series we examined 5 leiomyosarcomas and 2 intimal sarcomas. One intimal sarcoma was classified as malignant mesenchymoma of intimal origin because it showed fibroblastic and osteosarcomatous differentiation. However, Burke and Virmani pointed out that PA sarcomas frequently demonstrate osteosarcomatous or chondrosarcomatous differentiation and should not be classified as mesenchymoma, unless there are areas of liposarcoma, myosarcoma, or angiosarcoma as well. Other reports mentioned that approximately $50 \%$ of PA sarcomas are of fibroblastic or myofibroblastic origin, whereas $20 \%$ are leiomyosarcomas. Another $30 \%$ of the cases were subclassified. ${ }^{13}$ Although previous immunohistochemical studies have aided in the subclassification of great vessel sarcomas, ${ }^{14}$ it is not possible to date to definitely determine the cell of tumor origin by immunohistochemical findings.

In patients with advanced PA sarcoma and significant vascular obstruction, surgical treatment offers adequate palliation and increases length of survival. ${ }^{6,15-17}$ Aggressive surgical resection may even be curative in patients with localized lesions and early diagnosis. Depending on tumor localization, distal extension, and the presence of pulmonary metastases, surgical therapy includes tumor and PA resection and reconstruction, endarterectomy techniques, and pneumonectomy. Because tumor lesions are usually originating from the central parts of the PA or the pulmonary valve, the use of cardiopulmonary bypass seems to be essential in all cases. In 6 of our 7 patients, tumor and PA resection was performed with additional endarterectomy in 4 and pneumonectomy in 2 patients. Because 1 of these patients has survived for more than 5 years without signs of tumor recurrence, we do prefer aggressive resection rather than endarterectomy alone whenever possible. Hypothermic circulatory arrest was used in 3 patients. One patient underwent a routine endarterectomy operation with circulatory arrest periods, and postoperative histology revealed the tumor growth within the thrombotic material. In 2 patients circulatory arrest was instituted after inadequate peripheral endarterectomy because of poor visibility under low-flow conditions. Because the frozen sections of the endarterectomy specimen in these 2 patients revealed unilateral peripheral positive margins, both patients received additional pneumonectomy, and 1 patient is alive 35 months after the operation, despite the presence of contralateral pulmonary metastases. Although the role of pneumonectomy in this context remains to be defined, it is a therapeutic option in combination with central PA resection when frozen sections reveal unilateral peripheral tumor spread.

Because median survival of these patients without surgical intervention is limited to approximately 1.5 months ${ }^{6}$ and quality of life is poor because of progressive right heart dysfunction, ${ }^{5}$ surgical treatment should be attempted in any case, even if cure seems unlikely. In this series there was no operative mortality and a significant improvement in exercise tolerance, and quality of life was achieved in all patients for months or years after operations. Although tumor recurrence $(n=4)$, pulmonary metastases $(n=4)$, or both, occurred in 6 of our 7 patients, the median length of survival could be increased to 19 months. On the basis of previous reports of 27 patients with surgical resection in the literature, Krüger and colleagues ${ }^{6}$ calculated a median survival of 10 months, with an early mortality of $22 \%$. Although sufficient data on adjuvant treatment are not available in the literature, neoadjuvant or adjuvant radiotherapy, chemotherapy, or both, in addition to resection, may play a role in an interdisciplinary approach and might increase the length of survival. ${ }^{5,18}$ Five of our 7 patients received postoperative chemotherapy, and 2 are alive 21 and 35 months, respectively, after their operations with partial remissions of pulmonary metastases or recurrent tumor after polychemotherapy. The only long-term survivor without tumor recurrence also had postoperative chemotherapy.

Primary PA sarcomas are extremely uncommon tumors. The diagnosis is difficult and usually delayed. Early diagnosis in patients with characteristic symptoms of pulmonary vascular obstruction can be improved by CT and MRI scanning. Early radical surgical resection with cardiopulmonary bypass probably 
presents the only opportunity for a potential cure. In extensive bilateral disease surgical therapy offers significant palliation and increases length of survival. The role of adjuvant or neoadjuvant treatment strategies will have to be defined. PA sarcoma need not necessarily be a fatal diagnosis.

\section{REFERENCES}

1. Mayer E, Dahm M, Hake U, Schmid FX, Pitton M, Kupferwasser I, et al. Mid-term results of pulmonary thromboendarterectomy for chronic thromboembolic pulmonary hypertension. Ann Thorac Surg 1996;61:1788-92.

2. Mayer E, Kramm T, Dahm M, Moersig W, Eberle B, Duber C, et al. Early results of pulmonary thromboendarterectomy in chronic thromboembolic pulmonary hypertension. Z Kardiol 1997; 86:920-7.

3. Murthy MS, Meckstroth CV, Merkle BH, Huston JT, Cattaneo SM. Primary intimal sarcoma of pulmonary valve and trunk with osteogenic sarcomatous elements: report of a case considered to be pulmonary embolus. Arch Pathol Lab Med 1976;100:649-51.

4. Bleisch VR, Kraus FT. Polypoid sarcoma of the pulmonary trunk: analysis of the literature and report of a case with leptomeric organelles and ultrastructural features of rhabdomyosarcoma. Cancer 1980;46:314-24.

5. Parish JM, Rosenow EC 3rd, Swensen SJ, Crotty TB. Pulmonary artery sarcoma: clinical features. Chest 1996;110:1480-8.

6. Kruger I, Borowski A, Horst M, de Vivie ER, Theissen P, GrossFengels W. Symptoms, diagnosis, and therapy of primary sarcomas of the pulmonary artery. Thorac Cardiovasc Surg 1990;38:91-5.

7. Kauczor HU, Schwickert HC, Mayer E, Kersjes W, Moll R, Schweden F. Pulmonary artery sarcoma mimicking chronic thromboembolic disease: computed tomography and magnetic resonance imaging findings. Cardiovasc Intervent Radiol 1994;17:185-9.

8. Fasse A, Kauczor HU, Mayer E, Kreitner KF, Heussel CP, Thelen M. Sarcoma of the pulmonary artery-pre- and postoperative radiologic findings in initial tumor manifestation and recurrence. Rofo Fortschr Geb Rontgenstr Neuen Bildgeb Verfahr 1999;170:112-8.

9. Weinreb JC, Davis SD, Berkmen YM, Isom W, Naidich DP. Pulmonary artery sarcoma: evaluation using Gd-DTPA. J Comput Assist Tomogr 1990;14:647-9.

10. Rafal RB, Nichols JN, Markisz JA. Pulmonary artery sarcoma: diagnosis and postoperative follow-up with gadolinium-diethylenetriamine pentaacetic acid-enhanced magnetic resonance imaging. Mayo Clin Proc 1995;70:173-6.

11. Winchester PA, Khilnani NM, Trost DW, Litvak B, Gold JP, Sos TA. Endovascular catheter biopsy of a pulmonary artery sarcoma. AJR Am J Roentgenol 1996;167:657-9.

12. Burke AP, Virmani R. Sarcomas of the great vessels: a clinicopathologic study. Cancer 1993;71:1761-73.

13. Nonomura A, Kurumaya H, Kono N, Nakanuma Y, Ohta G, Terahata S, et al. Primary pulmonary artery sarcoma: report of two autopsy cases studied by immunohistochemistry and electron microscopy, and review of 110 cases reported in the literature. Acta Pathol Jpn 1988;38:883-96.

14. McGlennen RC, Manivel JC, Stanley SJ, Slater DL, Wick MR, Dehner LP. Pulmonary artery trunk sarcoma: a clinicopathologic, ultrastructural, and immunohistochemical study of four cases. Mod Pathol 1989;2:486-94.

15. Anderson MB, Kriett JM, Kapelanski DP, Tarazi R, Jamieson SW. Primary pulmonary artery sarcoma: a report of six cases. Ann Thorac Surg 1995;59:1487-90.

16. Tanaka I, Masuda R, Inoue M, Kasahara D, Furuhata Y, Shimizu $\mathrm{S}$, et al. Primary pulmonary-artery sarcoma: report of a case with complete resection and graft replacement, and review of 47 surgically treated cases reported in the literature. Thorac Cardiovasc Surg 1994;42:64-8.

17. Iversen S, Hake U, Schmiedt W, Jakob H, Ramp U, Gabbert H, et al. Resection of central primary pulmonary artery sarcoma. Eur J Cardiothorac Surg 1991;5:603-7.

18. Zerkowski HR, Hofmann HS, Gybels I, Knolle J. Primary sarcoma of pulmonary artery and valve: multimodality treatment by chemotherapy and homograft replacement. J Thorac Cardiovasc Surg 1996;112:1122-4. 